\title{
F-actin cross-linking enhances the stability of force generation in disordered actomyosin networks
}

\author{
Wonyeong Jung ${ }^{1}$ - Michael P. Murrell ${ }^{2,3}$ - Taeyoon Kim ${ }^{4}$
}

Received: 29 December 2014 / Revised: 12 May 2015 / Accepted: 30 May 2015 / Published online: 10 June 2015

(C) OWZ 2015

\begin{abstract}
Myosin molecular motors and actin cross-linking proteins (ACPs) are known to mediate the generation and transmission of mechanical forces within the cortical F-actin cytoskeleton that drive major cellular processes such as cell division and migration. However, how motors and ACPs interact collectively over diverse timescales to modulate the time-dependent mechanical properties of the cytoskeleton remains unclear. In this study, we present a three-dimensional agent-based computational model of the cortical actomyosin network to quantitatively determine the effects of motor activity and the density and kinetics of ACPs on the accumulation and maintenance of mechanical tension within a disordered actomyosin network. We found that motors accumulate large stress quickly by behaving as temporary cross-linkers although this stress is relaxed over time unless there are sufficient passive ACPs to stabilize the network. Stabilization by ACPs helps motors to generate forces up to their maximum potential, leading to significant enhancement of the efficiency and stability of stress generation. Thus, we demonstrated that the force-dependent kinetics of ACP
\end{abstract}

Electronic supplementary material The online version of this article (doi:10.1007/s40571-015-0052-9) contains supplementary material, which is available to authorized users.

$\triangle$ Taeyoon Kim

kimty@purdue.edu

1 School of Mechanical Engineering, Purdue University, 585 Purdue Mall, West Lafayette, IN 47907, USA

2 Department of Biomedical Engineering, Yale University, 55 Prospect Street, New Haven, CT 06520, USA

3 Systems Biology Institute, Yale University, 840 West Campus Drive, West Haven, CT 06516, USA

4 Weldon School of Biomedical Engineering, Purdue University, 206 S Martin Jischke Drive, West Lafayette, IN 47907, USA dissociation plays a critical role for the accumulation and sustainment of stress and the structural remodeling of networks.

Keywords Actin - Myosin - Actomyosin - Cortex · Simulation

\section{Introduction}

Living cells utilize mechanical forces for critical biological functions such as cytokinesis, cell migration, and muscle contraction [1]. Actomyosin contractility is responsible for generating tensile forces in muscle, smooth muscle, and nonmuscle cells, leading to various morphological changes from subcellular to tissue length scales [2-4]. The cell cortex is a thin network of actin filaments (F-actins), cross-linked by actin cross-linking proteins (ACPs) and decorated by myosin molecular motors, whose local contractility drives the growth of spherical membrane protrusion called blebs and largescale contractility facilitates the detachment and retraction of cell body during migration [5]. Tight regulation of cortical contractility is crucial for the definition and maintenance of polarization axis during cell division [6]. The cortex often undergoes dramatic structural remodeling due to F-actin turnover and myosin-mediated contractions, which enables cells to survive in rapidly changing extracellular environments [7]. Thus, understanding how the cell cortex generates forces and structurally remodels is a prerequisite for elucidating the underlying mechanisms of biological processes including migration and division.

A wide variety of in vitro reconstitution experiments have provided insights into the understanding of actomyosin contractility. Initial studies recombined purified F-actins, myosins, and ACPs to identify the minimal prerequisites for 
contractility and structural reorganization of disorganized actomyosin networks. As a result, it has been established that the dynamics and extent of contractility are regulated by F-actin network architecture [8], as well as the density and interplay of myosin motors and ACPs [9-14]. From these studies, it was found that the contractility arises from a symmetry breaking between tensile and compressive forces via buckling of F-actin $[15,16]$. In addition, it has been shown that molecular motors can tune the viscoelastic properties of networks. For example, mechanical stress arising from molecular motor and ACP activities significantly increases the stiffness of actomyosin networks by more than two orders of magnitude in an ATP-dependent manner [17,18].

Concurrently, various computational and theoretical approaches have been developed to study force generation in actomyosin networks. The origin of force generation has been associated with local deformations of F-actin which result in net tension on networks by breaking symmetry between tensile and compressive forces [19,20]. By contrast, the propensity of motors to reposition toward lower energy configurations can also lead to net tensile stress [21]. The force generation can lead to changes in the structural organization of the F-actin network. At elevated motor density, contractile forces drive the formation of bundles from disordered networks [22]. In the presence of ACPs, motor stresses drive initially well-connected networks to a critical state with the formation of clusters and rupture of ACPs, or stabilize initially floppy networks, enhancing network stiffness [23-25].

However, to date, few computational models have quantitatively described the time-variant contractile behaviors of cortical actomyosin networks or have faithfully captured the detailed geometry and mechanochemistry of myosin thick filaments and the mechanics and kinetics of F-actins and ACPs. To provide new insights into the underlying mechanisms of force generation and structural remodeling, we developed a computational model for the thin cortex-like actomyosin networks clamped to an elastic substrate using explicit, benchmarked representation for myosin motors and ACPs. We first evaluated the maximum level and sustainability of stress generated by the networks as well as changes in network morphology over a wide range of densities of motors and ACPs. We found that motors are able to behave as temporary cross-linkers during initial stress generation, leading to larger maximum stress with higher motor density. However, the generated stress relaxes over time unless there are a sufficient amount of ACPs. We also showed that ACPs enable motors to exert forces close to their maximum potential, significantly enhancing the efficiency and stability of stress generation. In addition, we demonstrated that the frequency of detachment of ACPs from F-actin has a dramatic impact on the accumulation of network stress and long-term changes in network morphology. These findings are consistent with those that we found in a previous study using actomyosin bundles with randomly aligned F-actins [26]. Thus, the mechanisms that govern contractility and force generation are robust across diverse F-actin architecture and dimension.

\section{Methods}

We simulated thin actomyosin networks consisting of Factins, passive ACPs, and active myosin motors, using an approach in our recent work [26]. Descriptions about details of the approach can be found in the previous work as well as Supplementary Text and Table S1.

\subsection{Mechanics of F-actins, ACPs, and motors}

F-actins are modeled as a series of cylindrical segments with fixed polarity (barbed and pointed ends) whose length and diameter are 140 and $7 \mathrm{~nm}$ respectively, connected by elastic hinges (Fig. 1a). ACPs are modeled as pairs of cylindrical arms of $23.5 \mathrm{~nm}$ in length and $10 \mathrm{~nm}$ in diameter, connected to each other by elastic hinges. Motors mimic the structure of myosin bipolar thick filaments (TF) [27] with a relatively rigid backbone with symmetric polarity comprising a bare zone of $42 \mathrm{~nm}$ in length at center and multiple segments of $42 \mathrm{~nm}$ in length connected by hinges. Each endpoint of the backbone segment has two motor arms. The arms of ACPs and motors can bind to binding sites located every $7 \mathrm{~nm}$ on actin segments. Motions of segments representing F-actins, ACPs, and motors are governed by the Langevin equation with inertia neglected. The sum of deterministic forces and stochastic force determines velocity of each segment that is then used in the Euler integration scheme to update positions of segments. The deterministic forces include extensional, bending, and repulsive forces described by harmonic potentials.

\subsection{Dynamics of ACPs}

ACPs transiently cross-link pairs of F-actins without preference for angle of contact. ACPs unbind from F-actins as follows:

$k_{\mathrm{u}, \mathrm{ACP}}= \begin{cases}k_{\mathrm{u}, \mathrm{ACP}}^{0} \exp \left(\frac{\lambda_{\mathrm{u}, \mathrm{ACP}}\left|\vec{F}_{\mathrm{s}, \mathrm{ACP}}\right|}{k_{\mathrm{B}} T}\right) & \text { if } r \geq r_{0, \mathrm{ACP}} \\ k_{\mathrm{u}, \mathrm{ACP}}^{0} & \text { if } r<r_{0, \mathrm{ACP}}\end{cases}$

where $\vec{F}_{\mathrm{S}, \mathrm{ACP}}$ is an extensional force acting on an ACP arm, $k_{\mathrm{u}, \mathrm{ACP}}^{0}$ is the zero-force unbinding rate coefficient, $\lambda_{\mathrm{u}, \mathrm{ACP}}$ is the force sensitivity of unbinding, and $k_{\mathrm{B}} T$ is thermal 
(a)

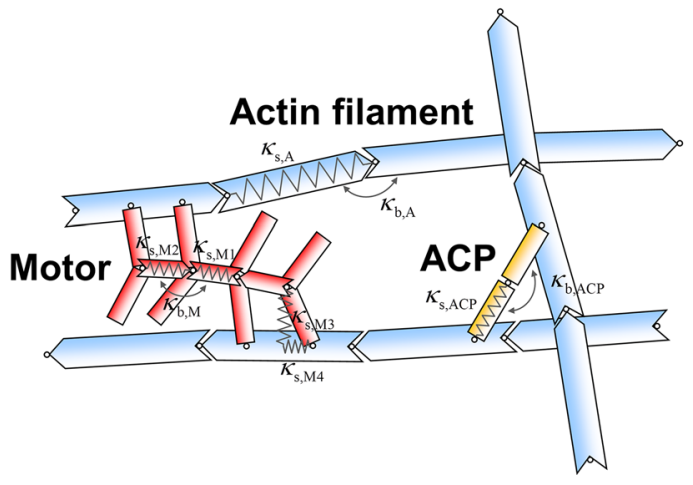

(b)

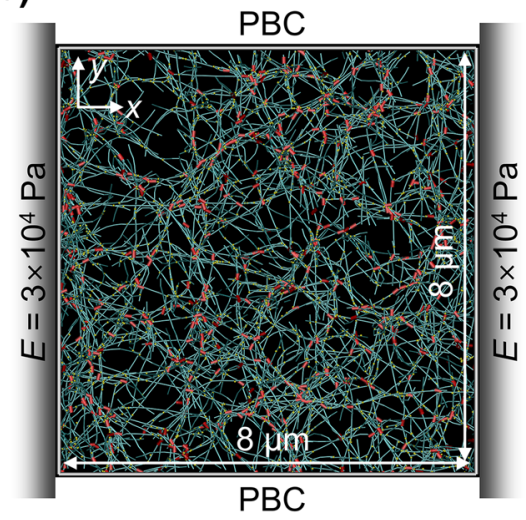

Fig. 1 Mechanics of actin filaments (F-actins), motors, and actin cross-linking proteins (ACPs) and computational setup for stress measurement. a F-actins consist of a series of cylindrical segments with barbed and pointed ends. ACPs comprise two arms connected in parallel. Motor thick filaments consist of a backbone structure with symmetric polarity, and each endpoint of the backbone segments contains two cylindrical arms. The arms of ACPs and motors bind to binding sites on F-actins. Several extensional $\left(\kappa_{\mathrm{S}}\right)$ and bending stiffnesses $\left(\kappa_{\mathrm{b}}\right)$ maintain equilibrium lengths and angles formed by segments of F-actins (A), motors (M), and ACPs. b A thin cortex-like network is clamped to the $x$ boundaries which behave as an elastic substrate with Young's modulus, $E$, set to $3 \times 10^{4} \mathrm{~Pa}$. Periodic boundary condition $(\mathrm{PBC})$ is applied in $y$ direction to simulate an infinitely large network

of ACPs. We used mechanochemical rates for motors which result in unloaded walking velocity of $\sim 140 \mathrm{~nm} / \mathrm{s}$ and stall force of $f_{\mathrm{M}}^{\text {stall }} \sim 5.7 \mathrm{pN}$.

\subsection{Network preassembly}

As in our previous study [32], cross-linked actomyosin networks are preassembled via self-assembly of actin monomers (G-actin), ACPs, and motors within a thin three-dimensional rectangular domain $(8 \times 8 \times 0.5 \mu \mathrm{m})$ with periodic boundary condition (PBC) in $\mathrm{x}$ and $\mathrm{y}$ directions. Two boundaries located in $\mathrm{z}$ direction exert repulsive forces only in the $\mathrm{z}$ direction to keep network elements within the domain. During the self-assembly, G-actins are polymerized into F-actins, and the TF structure of motors is formed via nucleation and polymerization of motor backbone segments with their arms binding to F-actin in the absence of walking events. Concurrently, ACPs also bind to F-actins, forming functional cross-links between pairs of F-actins. Then, the PBC is deactivated in the $\mathrm{x}$ direction, and Factins crossing the $\mathrm{x}$ boundaries are severed and irreversibly clamped to the $\mathrm{x}$ boundaries (Fig. 1b). No actin filaments are allowed to be clamped to the $\mathrm{x}$ boundaries during simulations. Unless specified, we used the following "reference" parameter values: initial G-actin concentration $\left(C_{\mathrm{A}}\right)$ is $25 \mu \mathrm{M}$, average F-actin length $\left(<L_{\mathrm{f}}>\right)$ is $\sim 1.44 \mu \mathrm{m}$, and the molar ratios of motors $\left(R_{\mathrm{M}}=C_{\mathrm{M}} / C_{\mathrm{A}}\right)$ and ACPs $\left(R_{\mathrm{ACP}}=C_{\mathrm{ACP}} / C_{\mathrm{A}}\right)$ are 0.014 and 0.1 , respectively. Note that $R_{\mathrm{M}}$ takes all $N_{\mathrm{a}} N_{\mathrm{h}}$ myosin heads into account. 


\subsection{Measurement of force, stress, and elastic moduli}

After the network preassembly, it is assumed that the $\mathrm{x}$ boundaries behave as an elastic substrate with Young's modulus, $E$, set to $3 \times 10^{4} \mathrm{~Pa}$ (Fig. 1b). Normal stress generated by a network over time is calculated by $\sigma(t)=E \varepsilon(t)$ where $\varepsilon(t)$ is the normal strain of the elastic substrate in $\mathrm{x}$ direction. We calculated the maximum $\left(\sigma_{\max }\right)$ and sustainability $\left(S=<\sigma>/ \sigma_{\max }\right)$ of the normal stress, where $<\sigma>$ indicates the time average of $\sigma(t)$ between a time point when $\sigma$ is $\sigma_{\max }$ and $t=100 \mathrm{~s}$.

We also evaluated microscopic forces acting on motors when $\sigma$ is close to $\sigma_{\max }, f_{\mathrm{M}}^{\max }=\vec{F}_{\mathrm{s}, \mathrm{M} 4} \cdot \vec{u} / N_{\mathrm{h}}$, and on ACPs, $f_{\mathrm{ACP}}^{\max }=\vec{F}_{\mathrm{s}, \mathrm{ACP}} \cdot \vec{u}$, where $\vec{F}_{\mathrm{s}, \mathrm{M} 4}$ is force acting on the spring governed by $\kappa_{\mathrm{s}, \mathrm{M} 4}$, and $\vec{u}$ is a unit vector in the axial direction of an actin segment toward a barbed end. Note that $f_{\mathrm{M}}^{\max }$ and $f_{\mathrm{ACP}}^{\max }$ are positive when acting toward barbed ends of F-actins. As shown later (Figs. $5 \mathrm{~b}, 6 \mathrm{~b}, 7 \mathrm{~b}$ ), $f_{\mathrm{M}}^{\max }$ is predominantly positive because $k_{\mathrm{u}, \mathrm{M}}$ becomes very large if $\vec{F}_{\mathrm{s}, \mathrm{M} 4}$ is directed toward a pointed end. By contrast, $f_{\mathrm{ACP}}^{\max }$ shows largely symmetric distribution but is biased slightly toward negative values since ACPs sustain positive $f_{\mathrm{M}}^{\max }$.

Frequency-dependent storage $\left(E^{\prime}\right)$ and loss moduli $\left(E^{\prime \prime}\right)$ are evaluated by measuring stress in response to a small sinusoidal strain $(5 \%)$ applied to the $+x$ boundary of the domain with the $-x$ boundary fixed in a similar way to that described in our previous study [33]. Phase delay between the applied strain and measured stress is calculated as $\tan ^{-1}\left(E^{\prime \prime} / E^{\prime}\right)$.

\subsection{Evaluation of changes in network morphology}

During force generation, actomyosin networks show changes in morphology including the spatial distribution of motors and network mesh size (Movies S1-S3). Spatial redistribution of motors is quantified by calculating the distribution of separation distances between a centroid position of each motor TF and that of its nearest neighbor. Predominance of distances close to zero in the distribution is indicative of motor aggregation because the zero distances originate from accumulation of motors in the same vicinity. Thus, we evaluated a temporal increase in the relative frequency of the smallest separation distance in distribution of the distances between the nearest neighbor motors to estimate time evolution of the motor aggregation in each simulation.

In addition, variations in network mesh size are indirectly estimated by approximating the density distribution of the network [34]. The domain of the network is divided to $400 \times 400$ pixels in $\mathrm{x}$ and $\mathrm{y}$ directions, and the intensity of each pixel is one if any actin segment is located on the pixel but zero if not. Then, distribution of the distances between the nearest neighbor non-zero pixels within each column $(400$ pixels) aligned in the y direction is calculated. If a network exhibits a homogeneous morphology, the distribution can be fitted well by an exponentially decreasing function. Smaller exponential decay constant is indicative of larger mesh size. We evaluated a temporal decrease in the decay constant in the distribution to estimate time evolution of network mesh size in each simulation. Note that the $\mathrm{z}$ position of actin segments is not reflected for this estimation, which can lead to underestimation of network mesh size. However, a relative change in network mesh size over time can still be captured.

\section{Results and discussion}

We explored factors that govern the generation of mechanical tension and the viscoelasticity of cortex-like actomyosin networks. First, we probed the time evolution of stress, $\sigma(t)$, and evaluated storage and loss moduli ( $E$ ' and $E$ ") with three different combinations of molar ratios of motors $\left(R_{\mathrm{M}}\right)$ and $\operatorname{ACPs}\left(R_{\mathrm{ACP}}\right)$.

At low motor activity and high cross-linking density $\left(R_{\mathrm{M}}=0.014\right.$ and $\left.R_{\mathrm{ACP}}=0.1\right), \sigma$ quickly reaches its maximum, $\sigma_{\max } \sim 600 \mathrm{~Pa}$, and then remains relatively constant (Fig. 2). The network remodels nominally with only small changes in distribution of mesh size, motors, and forces for $100 \mathrm{~s}$ (Fig. 3a and Movie S1). In this case, constant network morphology is concomitant with constant elasticity. $E$ ' shows a very weak power-law dependence on frequency, $\sim f^{0.09}$, while $E$ " is minimum at $1 \mathrm{~Hz}$ (Fig. 3 b), and the network becomes the most elastic with the smallest phase delay at $1 \mathrm{~Hz}$. (Fig. S2a). After $\sigma$ reaches $\sigma_{\max }, E$ ' and $E$ " change minimally over time.

By contrast, at low motor activity and low cross-linking density $\left(R_{\mathrm{M}}=0.014\right.$ and $\left.R_{\mathrm{ACP}}=0.01\right)$, both $\sigma_{\max }$ and the sustainability, $S$, are reduced (Fig. 2), and the network morphology drastically changes over time (Movie S2), resulting in very large meshes and severe coalescence of motors

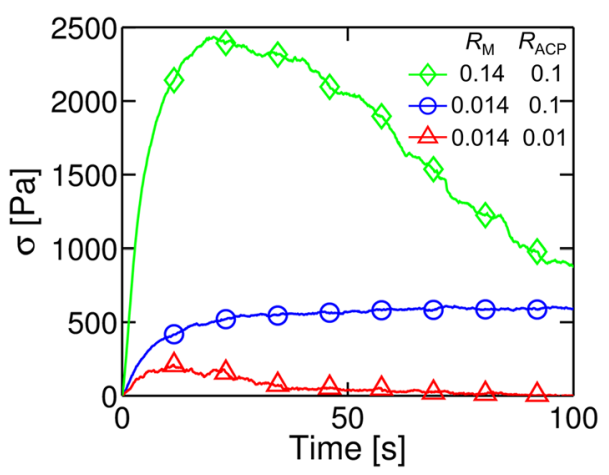

Fig. 2 Time evolution of stress, $\sigma(t)$, with three combinations of densities of motors $\left(R_{\mathrm{M}}\right)$ and $\mathrm{ACPs}\left(R_{\mathrm{ACP}}\right): R_{\mathrm{M}}=0.14$ and $R_{\mathrm{ACP}}=0.1$ (green diamonds), $R_{\mathrm{M}}=0.014$ and $R_{\mathrm{ACP}}=0.1$ (blue circles), and $R_{\mathrm{M}}=0.014$ and $R_{\mathrm{ACP}}=0.01$ (red triangles). (Color figure online) 
Fig. 3 Structural

reorganization correlates with

stiffening and softening of

networks. Network morphology

and viscoelastic moduli with

three combinations of $R_{\mathrm{M}}$ and

$R_{\mathrm{ACP}}$ used in Fig. 2. a, $\mathbf{c}, \mathbf{e}$

Distribution of distances

between each motor and its

closest neighbor and (inset)

distribution of mesh size of

networks measured at three time points. An increase in the relative frequency of the most left data point in the distribution of distances between the nearest neighbor motors indicates aggregation of motors. Note that distances are much smaller in (e) due to higher $R_{\mathrm{M}}$. Dashed lines in the insets show exponential fits to the distribution of mesh size, and a decrease in the exponential decay constant over time is indicative of an increase in mesh size. b, d, f Storage, $E^{\prime}$, and loss moduli, $E$ ", measured at three time ranges. A legend in (b) is shared with (d) and (f). Cyan inverted triangles show $E$, and $E$ " in the absence of ACP unbinding. (Color figure online) (a)

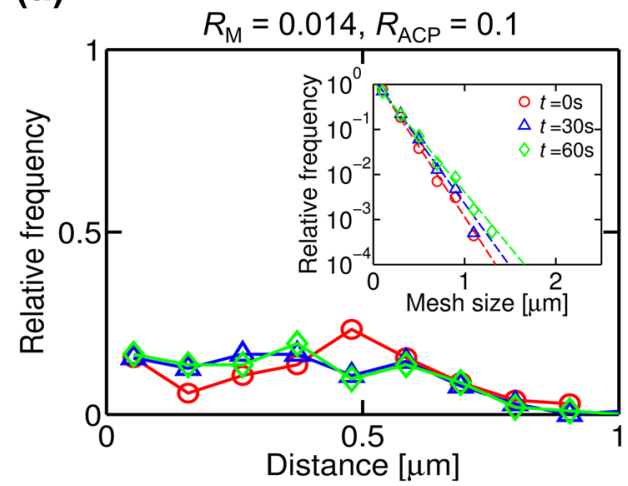

(c)

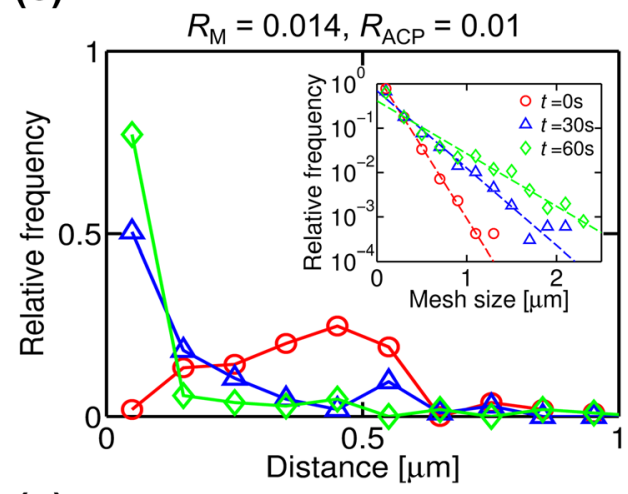

(e)

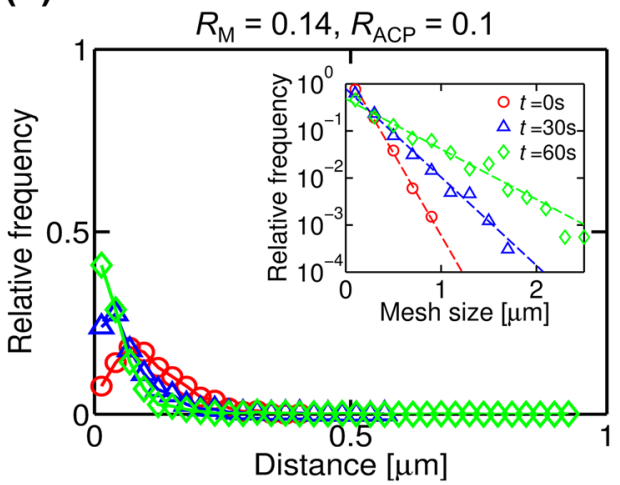

(b)

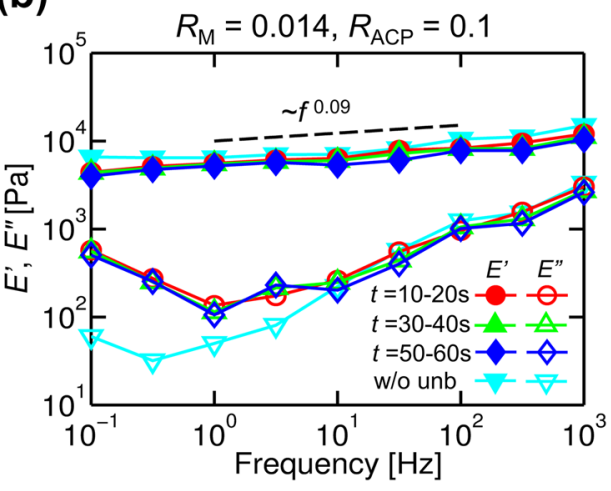

(d)

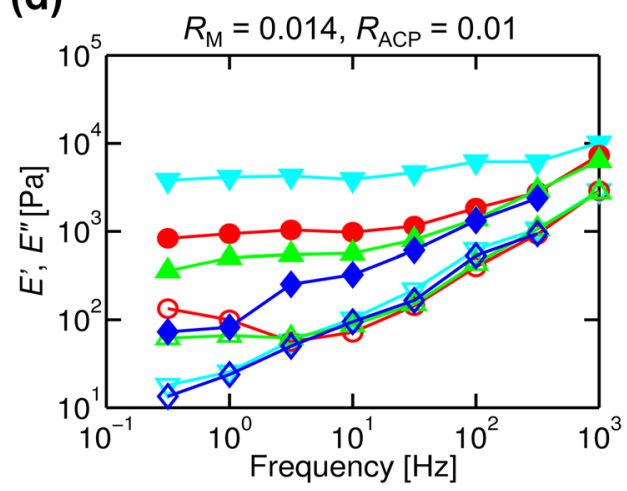

(f)

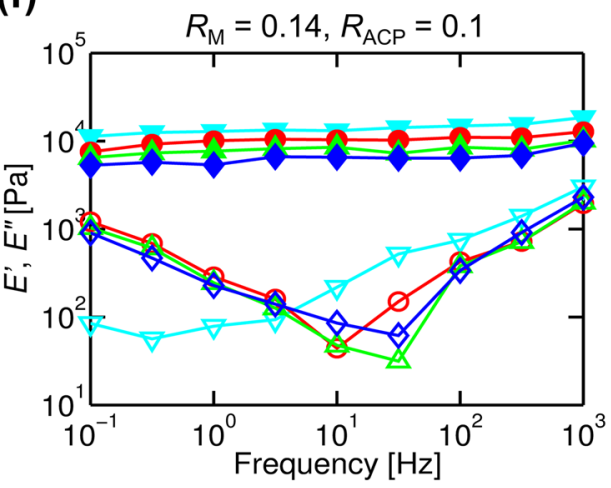

(Fig. 3c). At the end, the stress becomes nearly zero with emergence of separated aggregates. At $\sigma \sim \sigma_{\max }, E$ ' is much smaller but still shows the weak power-law dependence at low frequencies (Fig. 3d) with the minimal phase delay at $3.16 \mathrm{~Hz}$. As $\sigma$ diminishes over time, the network exhibits a large reduction in $E$ ' with greater power-law exponent while $E$ " remains relatively constant, resulting in greater phase delay (Fig. S2b). The rise of $E$ " at low frequencies gradually disappears over time. Thus, network remodeling modulated by ACPs is inversely related to network elasticity and to the ability to sustain mechanical stress.

With high motor activity and high cross-linking density ( $R_{\mathrm{M}}=0.14$ and $R_{\mathrm{ACP}}=0.1$ ), the generated stress is destabilized but shows significantly enhanced $\sigma_{\max }$ (Fig. 2). In addition, the accumulation of stress is accompanied by grad- ual coarsening of the network with increasing mesh size and aggregation of motors (Fig. 3e and Movie S3). E' is very high and nearly independent of frequency, showing a slow decrease over time (Fig. 3f), and the phase delay becomes minimal at $10-31.6 \mathrm{~Hz}$ (Fig. S2c).

Overall, the elasticity of the network tensed by motor activity is strongly dependent upon the kinetics of ACP unbinding. Without unbinding of ACPs, $E^{\prime}$ is high with very weak frequency dependence and hardly changes over time, and $E$ " shows a much smaller increase at low frequencies in all the three cases (Fig. 3b, d, f). This indicates that the temporal decrease in $E$ ' occurring with ten-fold higher $R_{\mathrm{M}}$ or ten-fold lower $R_{\mathrm{ACP}}$ and the existence and variation of the critical frequency are associated with the ACP unbinding. 
(a)

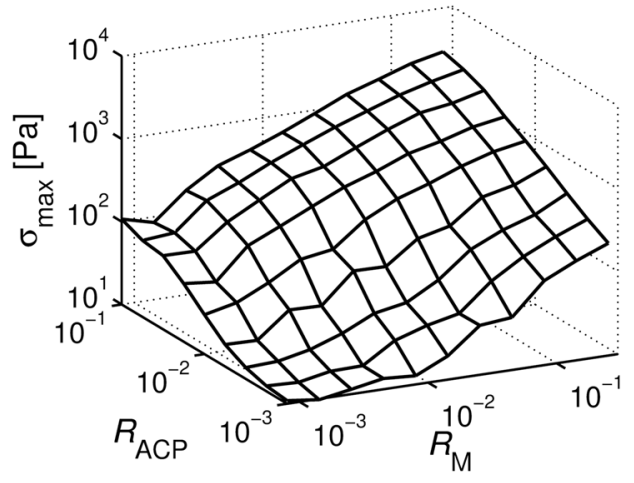

(c)

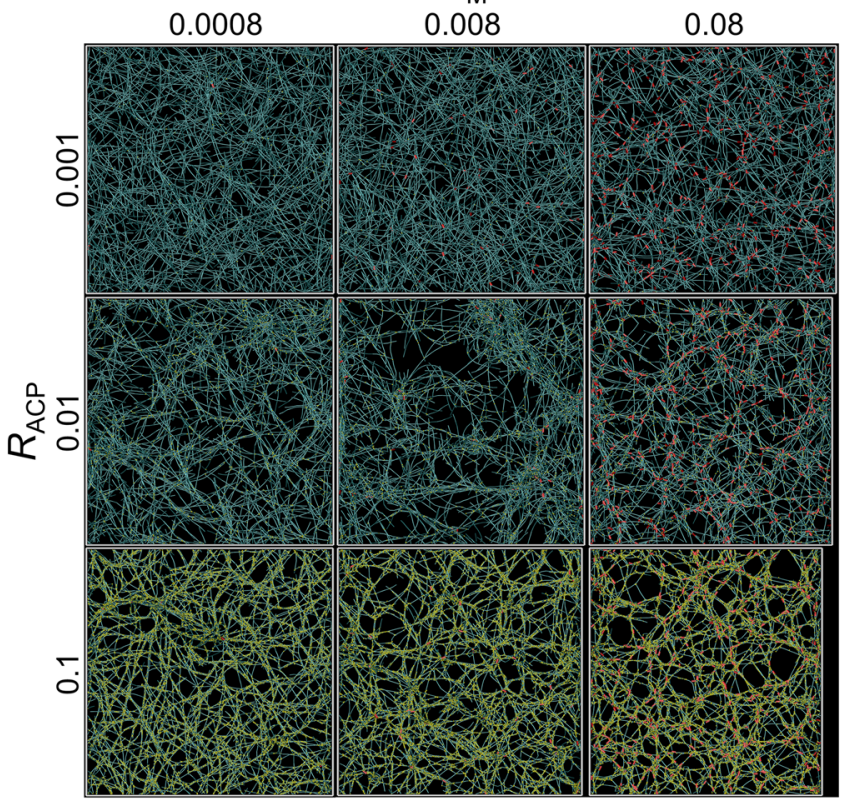

Actin, ACP, motor

Fig. 4 F-actin cross-linking and motor activity synergistically increase the magnitude and sustainability of mechanical stress. Effects of $R_{\mathrm{M}}$ and $R_{\mathrm{ACP}}$ on $\mathbf{a}, \mathbf{b}$ the maximum, $\sigma_{\max }$, and sustainability of the stress, $S$, and on $\mathbf{c}, \mathbf{d}$ the morphology of networks at $\sigma \sim \sigma_{\max }$. In (c), F-actins,

Interestingly, $E$, and $E^{\prime \prime}$ are closely related to $\sigma . E^{\prime}$ at a low frequency $(1 \mathrm{~Hz})$ is directly proportional to $\sigma$ at high $\sigma$ but becomes independent of $\sigma$ at low $\sigma$ as found in other study [25] (Fig. S2d). In the three cases tested above, exponent of the power-law dependence of $E$ ' on frequency is shown to be inversely proportional to $\sigma$. $E$ " at high frequencies is relatively the same regardless of $R_{\mathrm{M}}$ and $R_{\mathrm{ACP}}$, whereas the critical frequency at which a network becomes the most elastic tends to be higher when $\sigma$ is not sustained well (i.e. low $S$ ). All these are consistent with characteristics of frequency-dependent shear moduli, $G^{\prime}$ and $G^{\prime}$, in a previous experiment [18]. Therefore, $E^{\prime}$ and $E^{\prime \prime}$ are roughly predictable if $\sigma_{\max }$ and $S$ are known. (b)

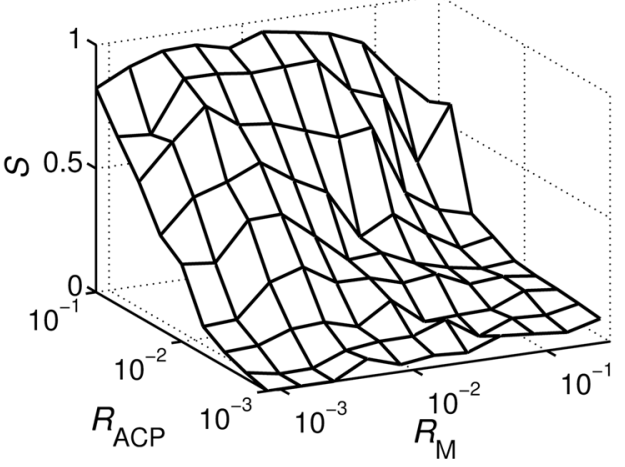

(d)

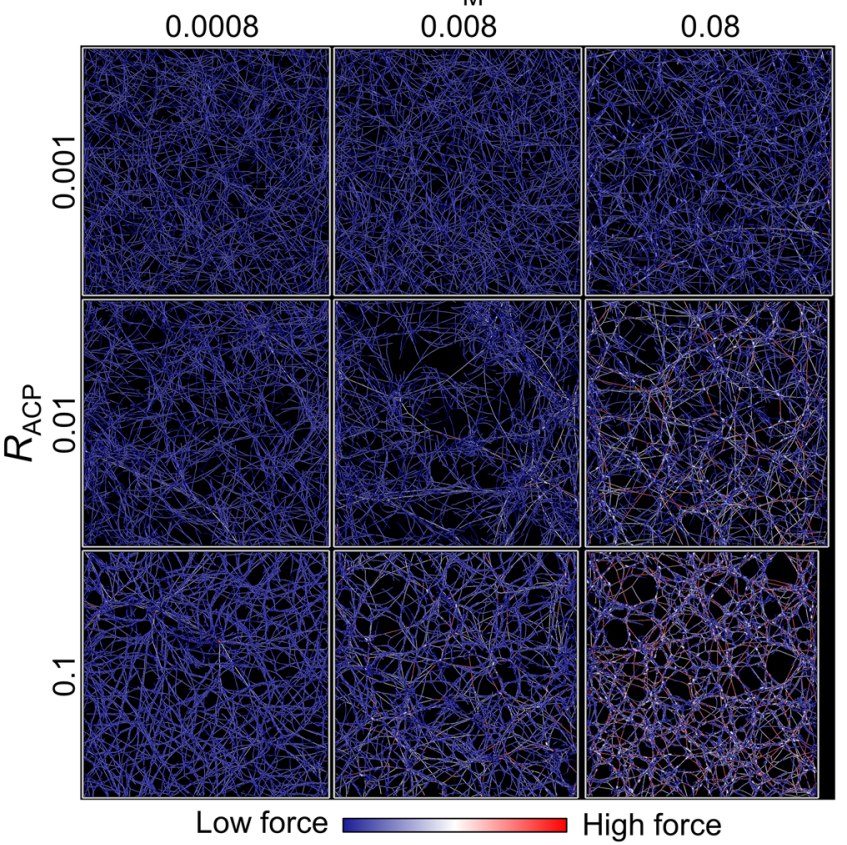

ACPs, and motors are indicated by cyan, yellow, and red, respectively. In (d), level of forces exerted on each constituent is represented by the color scaling. (Color figure online)

\subsection{Densities of motors and ACPs govern stress generation and network morphology}

To find a quantitative relationship between the molar ratios and the generated stress, we systematically evaluated $\sigma_{\max }$ and $S$ over a wide range of $R_{\mathrm{M}}$ and $R_{\mathrm{ACP}}$ (Fig. $4 \mathrm{a}, \mathrm{b}$ ), and visualized network morphology and force distribution when $\sigma$ reaches $\sigma_{\max }$ (Fig. 4c, d). Overall, $\sigma_{\max }$ increases with proportionality to both $R_{\mathrm{M}}$ and $R_{\mathrm{ACP}}$, and $S$ tends to be greater with smaller $R_{\mathrm{M}}$ and larger $R_{\mathrm{ACP}}$. A similar level of $\sigma_{\max }$ can be acquired with much fewer motors if a sufficient number of ACPs exist in a network, indicative of complementary effects of motors and ACPs on $\sigma_{\max }$ (Fig. 4a). However, $S$ of cases with similar $\sigma_{\max }$ can be quite different (Fig. 4b), so 
Fig. 5 Motor activity promotes stress generation and network remodeling but is antagonistic to stress sustainability. At

$R_{\mathrm{ACP}}=0.1$, influences of $R_{\mathrm{M}}$ on $\mathbf{a} \sigma_{\max }$ (red circles) and $S$ (blue triangles), $\mathbf{b}$ distribution of forces acting on motors $\left(f_{\mathrm{M}}^{\max }\right)$ and $\mathrm{ACPs}\left(f_{\mathrm{ACP}}^{\max }\right), \mathbf{c}$ a decrease in the decay constant of an exponential fit to the distribution of mesh size at $\sigma \sim$ $\sigma_{\max }$ or $t=100 \mathrm{~s}$, compared to that of the fit at $t=0 \mathrm{~s}$, and $\mathbf{d}$ an increase in the relative frequency of the most left data point in the distribution of distances between the nearest neighbor motors at $\sigma \sim \sigma_{\max }$ or $t=100 \mathrm{~s}$, compared to that at $t=$ $0 \mathrm{~s}$. In (c), a larger decrease in the decay constant represents a larger increase in network mesh size, and in (d), a larger increase in the relative frequency is indicative of more severe motor aggregation. (Color figure online)

Fig. 6 F-actin cross-linking increases stress generation and sustainability concomitantly but is antagonistic to network remodeling. At $R_{\mathrm{M}}=0.014$, impact of $R_{\mathrm{ACP}}$ on a $\sigma_{\max }$ (red circles) and $S$ (blue triangles), b distribution of $f_{\mathrm{M}}^{\max }$ and $f_{\mathrm{ACP}}^{\max }$, c a decrease in the decay constant of an exponential fit to the distribution of mesh size, and $\mathbf{d}$ an increase in the relative frequency of the most left data point in the distribution of distances between the nearest neighbor motors at $\sigma \sim \sigma_{\max }$ or $t=100 \mathrm{~s}$, compared to those at $t=0 \mathrm{~s}$. (Color figure online) (a)

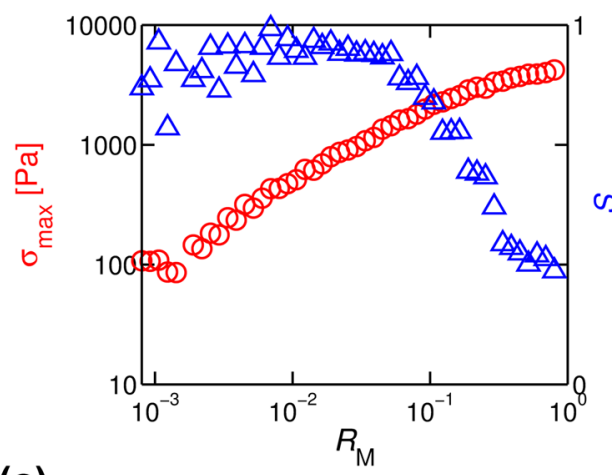

(c)

(b)

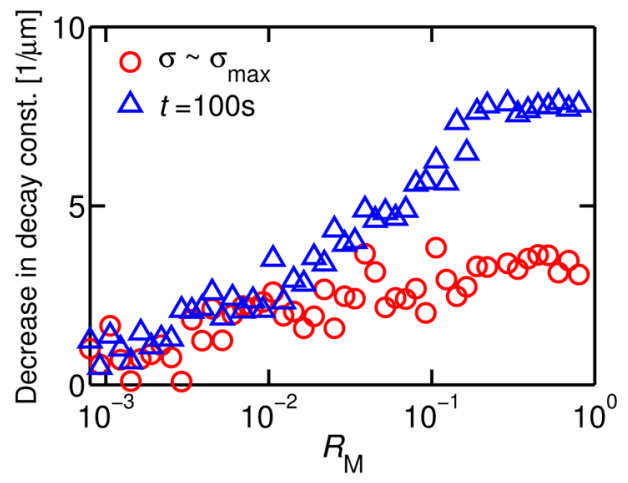

(d)
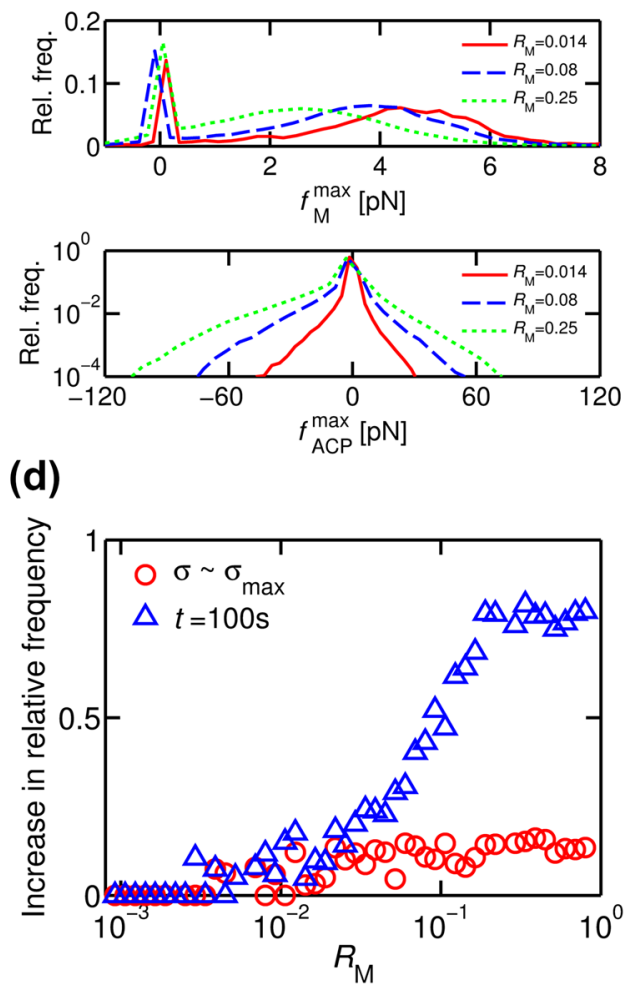

(a)

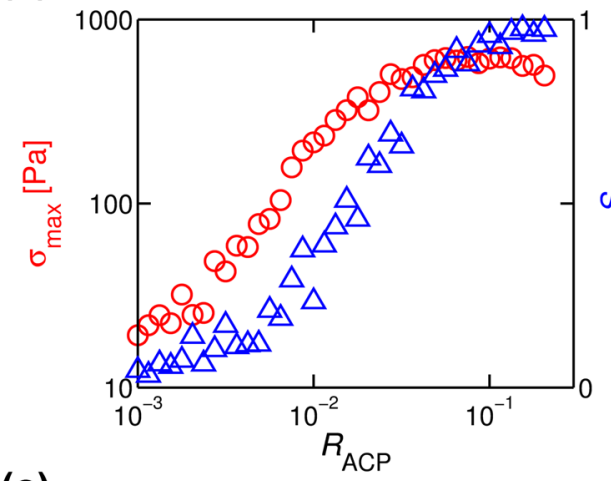

(b)
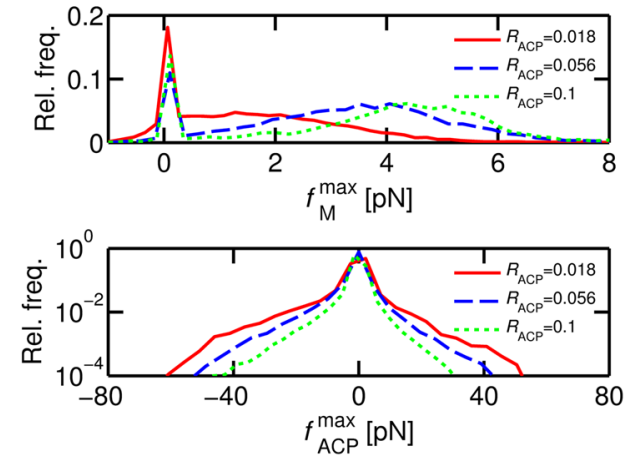

(d)
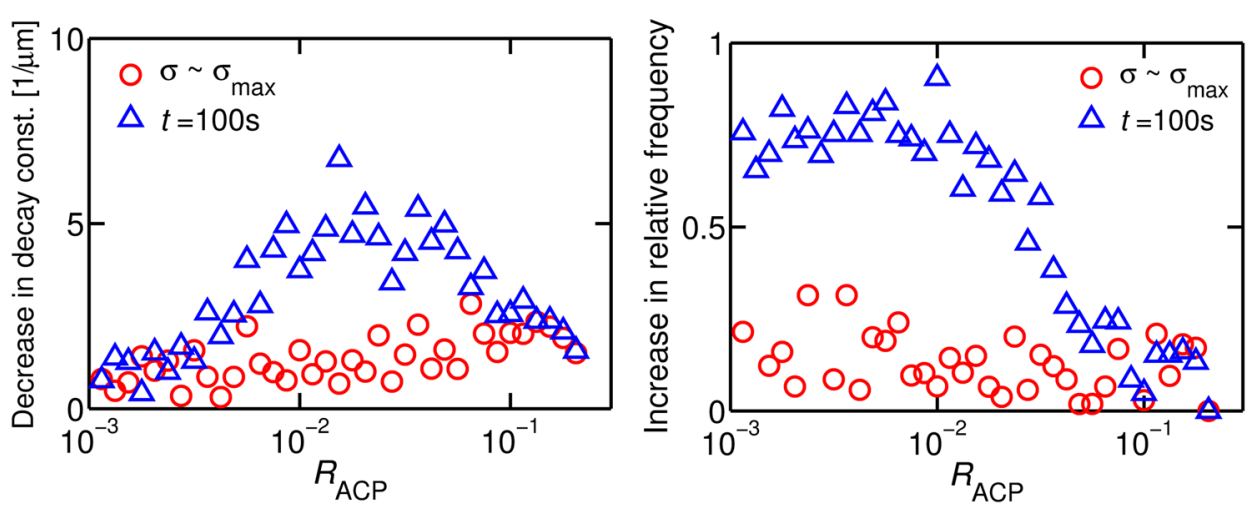
Fig. 7 ACP unbinding decreases stress accumulation and promotes network remodeling. At the fixed levels of $R_{\mathrm{M}}=0.014$ and $R_{\mathrm{ACP}}=0.1$, effects of zero-force unbinding rate coefficient of ACPs $\left(k_{\mathrm{u}, \mathrm{ACP}}^{0}\right)$ on a $\sigma_{\max }($ red circles $)$ and $S$ (blue triangles), $\mathbf{b}$ distribution of $f_{\mathrm{M}}^{\max }$ and $f_{\mathrm{ACP}}^{\max }$, a decrease in the decay constant of an exponential fit to the distribution of mesh size, and $\mathbf{d}$ an increase in the relative frequency of the most left data point in the distribution of distances between the nearest neighbor motors at $\sigma \sim \sigma_{\max }$ or $t=100 \mathrm{~s}$, compared to those at $t=0 \mathrm{~s}$. In (b), values in the legend show $k_{\mathrm{u}, \mathrm{ACP}}^{0} / k_{\mathrm{u}, \mathrm{ACP}}^{0 *}$ in each case. (Color figure online) (a)

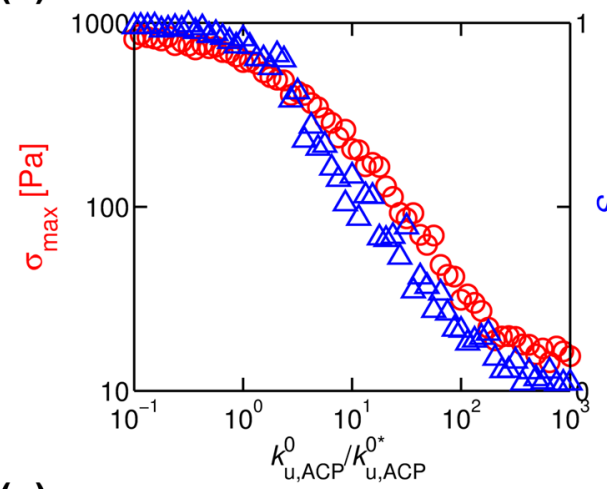

(c)

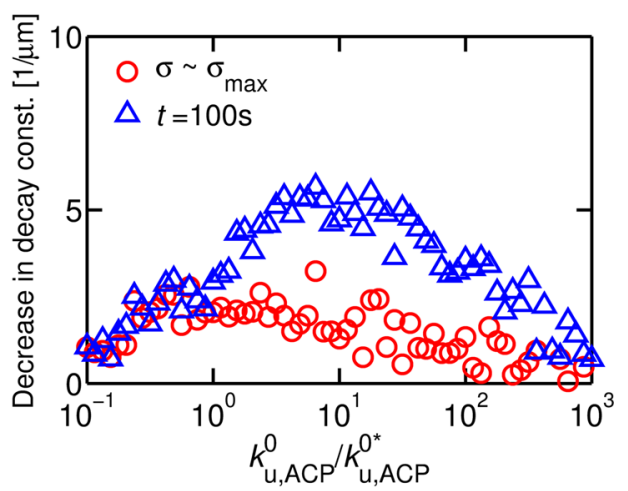

(b)
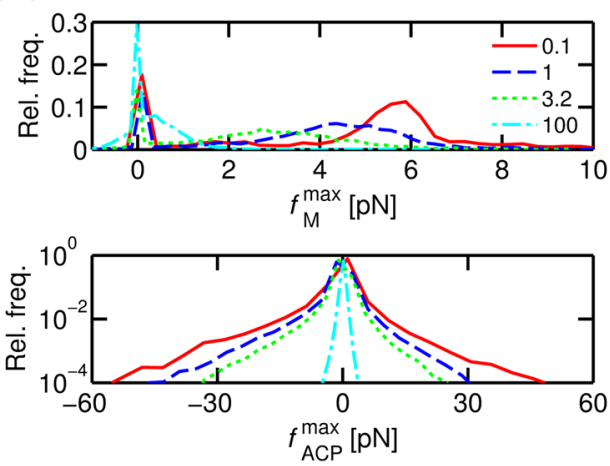

(d)

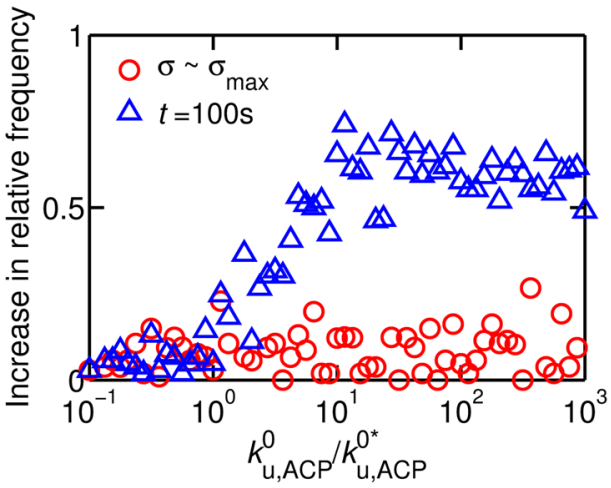

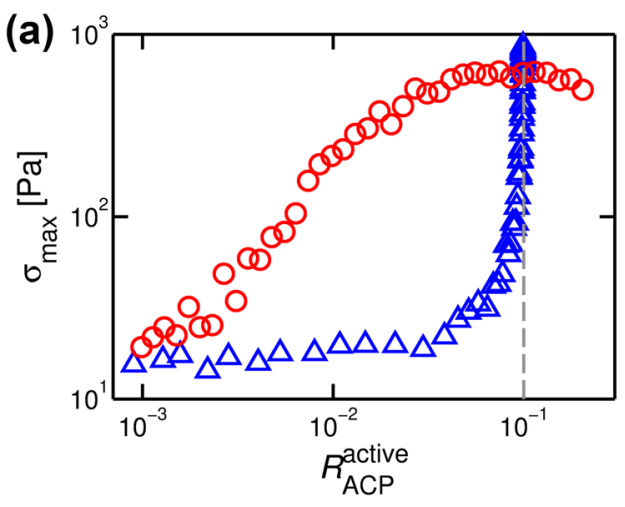

Fig. 8 Increased ACP unbinding disturbs stress accumulation effectively rather than merely changes the molar ratio of ACPs in the active state $\left(R_{\mathrm{ACP}}^{\text {active }}\right)$ at a dynamic equilibrium. Effects of $R_{\mathrm{ACP}}^{\text {active }}$ on a $\sigma_{\max }$ and $\mathbf{b} S$. $R_{\mathrm{ACP}}^{\text {active }}$ is altered by either varying $R_{\mathrm{ACP}}$ (red circles, data from

a relationship between $\sigma_{\max }$ and $S$ seems unclear. For better understanding about the relationship, we focused on how either $R_{\mathrm{M}}$ or $R_{\mathrm{ACP}}$ affects $\sigma_{\max }$ and $S$ with the other molar ratio fixed.

First, it was found that adding more motors at a constant level of $R_{\mathrm{ACP}}$ tends to increase $\sigma_{\max }$ but reduce $S$ with facilitation of network remodeling (Fig. 5). For example, at $R_{\mathrm{ACP}}$ $=0.1$ and low $R_{\mathrm{M}}, \sigma_{\max }$ increases in proportion to $R_{\mathrm{M}}$ following $\sigma_{\max } \sim R_{\mathrm{M}}^{0.6}$ with $S$ close to 1 (Fig. 5a), and network

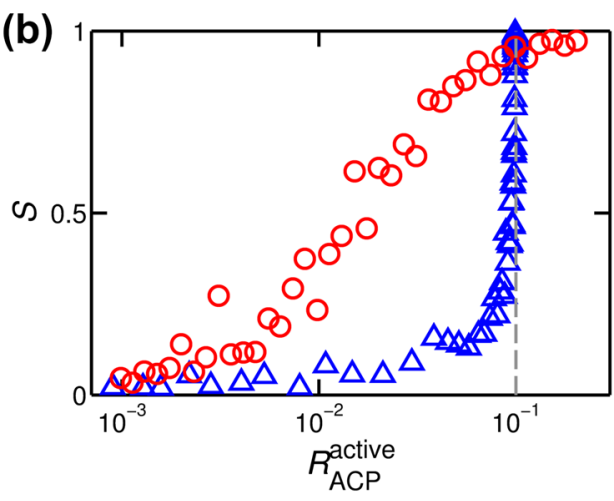

Fig. 6a) or $k_{\mathrm{u}, \mathrm{ACP}}^{0} / k_{\mathrm{u}, \mathrm{ACP}}^{0 *}$ (blue triangles, data from Fig. 7a). $R_{\mathrm{ACP}}^{\text {active }}$ is measured for $1 \mathrm{~s}$ before $\sigma(t)$ reaches $\sigma_{\max }$, and dashed lines indicate $R_{\mathrm{ACP}}^{\text {active }}=0.1$. (Color figure online)

morphology (i.e. mesh size and motor aggregation) hardly changes after $\sigma$ reaches $\sigma_{\max }$ (Fig. 5c, d and Supplementary Text). However, above $R_{\mathrm{M}} \sim 0.06, \sigma_{\max }$ slightly deviates from the power-law dependence, and $S$ rapidly drops with a decrease in $f_{\mathrm{M}}^{\max }$ and an increase in $f_{\mathrm{ACP}}^{\max }$ (Fig. 5b) as well as large variations in the network morphology over time. $\sigma_{\max } \sim R_{\mathrm{M}}^{0.6}$ at lower $R_{\mathrm{M}}$ and the variations at higher $R_{\mathrm{M}}-$ deviation from the power-law dependence and sharp drop of $S$-are consistent at lower $R_{\mathrm{ACP}}$ (Fig. S3a, b). Since $R_{\mathrm{ACP}}$ is 
fixed, a greater number of motors result in larger force acting on each ACP, which destabilizes bonds between ACPs and F-actins by increasing $k_{\mathrm{u}, \mathrm{ACP}}$ due to the force dependence described in Eq. 1. The destabilization prevents motors from exerting their maximum potential forces, $f_{\mathrm{M}}^{\text {stall }}$, and leads to lower $S$. It also facilitates large-scale remodeling of otherwise stable networks after $\sigma \sim \sigma_{\max }$.

Second, we found that addition of ACPs at a fixed level of $R_{\mathrm{M}}$ significantly increases $\sigma_{\max }$ and $S$ by helping motors to exert their stall forces but suppresses network remodeling (Fig. 6). For example, at $R_{\mathrm{M}}=0.014, \sigma_{\max }$ rapidly increases from $R_{\mathrm{ACP}}=0.001$ and reaches a plateau, $\sim 600$ $\mathrm{Pa}$, at $R_{\mathrm{ACP}} \sim 0.05$ (Fig. 6a). Existence of the plateau is attributed to the limited number of motors and their finite stall force as can be seen in various plateau levels depending on $R_{\mathrm{M}}$ (Fig. S3c). At $R_{\mathrm{M}}=0.014, S$ shows a sharp increase from $R_{\mathrm{ACP}} \sim 0.005$ and becomes nearly one at $R_{\mathrm{ACP}} \sim 0.1$. As the fixed level of $R_{\mathrm{M}}$ increases, $S$ begins to abruptly increase from higher $R_{\mathrm{ACP}}$ (Fig. $\mathrm{S} 3 \mathrm{~d}$ ). At $R_{\mathrm{M}}=0.014$ and $R_{\mathrm{ACP}}>$ 0.1 , the network morphology varies negligibly after $\sigma$ reaches $\sigma_{\max }$ (Fig. 6c, d and Supplementary Text). As above, with low $R_{\mathrm{ACP}}$, motors are incapable of exerting forces close to $f_{\mathrm{M}}^{\text {stall }}$ while $f_{\mathrm{ACP}}^{\mathrm{max}}$ is large (Fig. 6b). However, as $R_{\mathrm{ACP}}$ increases, $f_{\mathrm{M}}^{\max }$ rises to a level close to $f_{\mathrm{M}}^{\text {stall }}$, whereas $f_{\mathrm{ACP}}^{\max } \operatorname{dimin}$ ishes. Thus, with more ACPs, the force dependence of ACP unbinding is not likely to play an important role, stabilizing the bonds between ACPs and F-actins with higher $\sigma_{\max }$ and $S$ and with minimal network remodeling.

\subsection{Stability of ACPs determines long-time evolution of generated stress and morphology}

$\sigma_{\max }$ is proportional to both molar ratios since more motors provide greater force, and more ACPs can help the motors to exert their stall forces. For determining $\sigma_{\max }$, longterm stability of the bonds between ACPs and F-actins is not significant since it takes a relatively short time for $\sigma$ to reach $\sigma_{\max }$, and motors can contribute to enhancing connectivity temporarily as can be clearly seen in a difference of morphology and force distribution between a case with $R_{\mathrm{ACP}}=0.01$ and $R_{\mathrm{M}}=0.008$ and that with $R_{\mathrm{ACP}}=0.01$ and $R_{\mathrm{M}}=0.08$ (Fig. 4c, d) when $\sigma$ reaches $\sigma_{\max }$. Network remodeling at this early stage occurs minimally via local deformation of F-actins without large-scale network remodeling induced by numerous unbinding events of ACPs (Figs. 5c, d and 6c, d). By contrast, $S$ is proportional to $R_{\mathrm{ACP}}$ but inversely proportional to $R_{\mathrm{M}}$ because the stability of the bonds is very crucial for high $S$. The long-term stability is highly affected by whether or not the force dependence of ACP unbinding is activated by large force exerted on ACPs. The magnitude of the force acting on ACPs is likely to be roughly proportional to the ratio of $R_{\mathrm{M}}$ to $R_{\mathrm{ACP}}$, which explains the relation between $S$ and the two molar ratios. Substantial network remodeling may emerge with large increases in mesh size and motor aggregation if the forceinduced destabilization of ACP bonds occurs. In such a case, major structural reorganization of networks occurs during relaxation of generated stress, not during stress generation.

\subsection{Kinetics of ACPs differentially regulates stress generation and network architecture}

As an increase in $k_{\mathrm{u}, \mathrm{ACP}}$ caused by the force sensitivity has the large effects, we found that an increase in the zero-force unbinding rate coefficient, $k_{\mathrm{u}, \mathrm{ACP}}^{0}$, reduces $\sigma_{\max }$ and $S$ but promotes the network remodeling (Fig. 7). $\sigma_{\max }$ and $S$ are less sensitive to $k_{\mathrm{u}, \mathrm{ACP}}^{0}$ at $k_{\mathrm{u}, \mathrm{ACP}}^{0}<k_{\mathrm{u}, \mathrm{ACP}}^{0 *}$ but become inversely proportional to $k_{\mathrm{u}, \mathrm{ACP}}^{0}$ at $k_{\mathrm{u}, \mathrm{ACP}}^{0}>k_{\mathrm{u}, \mathrm{ACP}}^{0 *}$ (Fig. 7a). The inverse proportionality of $\sigma_{\max }$ and $S$ to $k_{\mathrm{u}, \mathrm{ACP}}^{0}$ hardly changes despite a variation in $R_{\mathrm{M}}$ or $R_{\mathrm{ACP}}$ (Fig. S4). Dependence of $\sigma_{\max }, S$, and the network morphology on $k_{\mathrm{u}, \mathrm{ACP}}^{0}$ (Fig. 7a, c, d) seems opposite to that on $R_{\mathrm{ACP}}$ (Fig. 6a, $\mathrm{c}, \mathrm{d})$ because increases in $k_{\mathrm{u}, \mathrm{ACP}}^{0}$ and $R_{\mathrm{ACP}}$ have opposite effects on the molar ratio of ACPs in the active state, $R_{\mathrm{ACP}}^{\text {active }}$ (i.e. the number of ACPs bound to pairs of F-actins at a dynamic equilibrium). If a decrease in $R_{\mathrm{ACP}}^{\text {active }}$ is a sole outcome induced by an increase in $k_{\mathrm{u}, \mathrm{ACP}}^{0}, \sigma_{\max }$ and $S$ should be similar between cases with the same $R_{\mathrm{ACP}}^{\text {active }}$, regardless of how $R_{\mathrm{ACP}}^{\text {active }}$ is changed. In Fig. 8a, b, $\sigma_{\max }$ and $S$ of cases where $R_{\mathrm{ACP}}$ is varied (Fig. 6a) and of those where $k_{\mathrm{u}, \mathrm{ACP}}^{0}$ is changed (Fig. 7a) with respect to $R_{\mathrm{ACP}}^{\text {active }}$ are shown. A large difference in $\sigma_{\max }$ and $S$ between two groups at each $R_{\text {ACP }}^{\text {active was observed, implying that subsequent binding and }}$ unbinding of ACPs effectively dissipate elastic energy built by motors rather than merely reduce $R_{\mathrm{ACP}}^{\text {active }}$. Indeed, the effect of increasing $k_{\mathrm{u}, \mathrm{ACP}}^{0}$ on $f_{\mathrm{ACP}}^{\max }$ (Fig. 7b) is opposite to that of decreasing $R_{\mathrm{ACP}}$ (Fig. $6 \mathrm{~b}$ ) although both changes reduce $R_{\mathrm{ACP}}^{\text {active }}$. Higher $k_{\mathrm{u}, \mathrm{ACP}}^{0}$ would allow ACPs to sustain forces only for a short period before ACPs lose the forces due to unbinding, which deteriorates stress generation (low $\sigma_{\max }$ ) and maintenance (low $S$ ) but facilitates the network remodeling.

\subsection{ACPs help motors to generate the maximum force by inducing friction between $\mathrm{F}$-actins}

Dependence of $\sigma_{\max }$ and $S$ on $R_{\mathrm{M}}, R_{\mathrm{ACP}}$, and $k_{\mathrm{u}, \mathrm{ACP}}^{0}$ can be explained by local interactions between motors and ACPs. Motors tend to pull pairs of F-actins in opposite directions, which is capable of developing a mechanical force whose magnitude varies depending on how freely F-actins are displaced. If the F-actins are stably anchored to other F-actins by ACPs or clamped to boundaries, the motors will easily 
reach their stall force because force can quickly be built by their walking motion. By contrast, if the F-actins are unstably anchored or free to move, the F-actins can glide across motors without generation of large forces. Considering a small percentage of the clamped F-actins, the transient connection between F-actins via ACPs plays a critical role for helping motors to produce their maximum stall forces. The transience makes ACPs behave as a molecular clutch that transmits forces between pairs of F-actins via effective friction that is governed by $k_{\mathrm{u}, \mathrm{ACP}}^{0}$ and how much force is acting on each ACP at given values of $R_{\mathrm{M}}$ and $R_{\mathrm{ACP}}$. Long-term changes in network morphology accompanied by large meshes and severe motor aggregation may or may not occur, depending on whether or not the transient connection becomes unstable by the force acting on ACPs.

\subsection{Force-induced destabilization of ACPs also affects frequency-dependent viscoelastic moduli}

Initially, using three sets of $R_{\mathrm{M}}$ and $R_{\mathrm{ACP}}$, we showed that the critical frequency where a network becomes the most elastic (i.e. the lowest phase delay) can shift to higher frequencies by either increasing $R_{\mathrm{M}}$ or decreasing $R_{\mathrm{ACP}}$, and that the local minimum of $E$ " disappears if ACPs do not unbind (Fig. 3b, d, f). In other studies, it has been shown that the critical frequency represents the transition point below which ACPs begin to behave as transient cross-linkers and therefore elevate loss modulus, and that the critical frequency can vary or disappear by tuning unbinding rates of ACPs $[35,36]$. The shift of the critical frequency observed in our results can be explained by force-induced acceleration of ACP unbinding. As the ratio of $R_{\mathrm{M}}$ to $R_{\mathrm{ACP}}$ becomes greater by ten-fold higher $R_{\mathrm{M}}$ or ten-fold lower $R_{\mathrm{ACP}}, k_{\mathrm{u}, \mathrm{ACP}}$ dramatically increases due to large forces acting on ACPs, resulting in the shift of the critical frequency to higher frequencies. This is why the increase in the critical frequency is associated with low $S$ that is also caused by the force-induced destabilization.

\subsection{Universality of the mechanisms of force generation occurring within actomyosin units}

Contractile actomyosin structures in non-muscle cells vary in terms of the polarity, length, density, and orientation of F-actins. We previously found that in actomyosin bundles with randomly aligned F-actins, the balance between motors and ACPs determines the generation of mechanical forces [26]. Surprisingly, force generation in cortex-like networks which contain additional disorder in $\mathrm{F}$-actin orientation as well as different local F-actin density is driven by similar mechanisms. We also found that a three-dimensional cubical network $(3 \times 3 \times 3 \mu \mathrm{m})$ exhibits similar dependences of $\sigma_{\max }$ and $S$ on $R_{\mathrm{M}}$ and $R_{\mathrm{ACP}}$ (Fig. S5), which demonstrates the universality of the mechanisms found in this study, regardless of dimensionality of actomyosin structures.

Acknowledgments We gratefully acknowledge the support of the National Science Foundation (1434013-CMMI and 1434095-CMMI).

\section{References}

1. Tee SY, Bausch AR, Janmey PA (2009) The mechanical cell. Curr Biol 19(17):R745-R748

2. Kee AJ, Gunning PW, Hardeman EC (2009) Diverse roles of the actin cytoskeleton in striated muscle. J Muscle Res Cell Motil 30(56): $187-197$

3. Vicente-Manzanares M, Ma X, Adelstein RS, Horwitz AR (2009) Non-muscle myosin II takes centre stage in cell adhesion and migration. Nat Rev Mol Cell Biol 10(11):778-790

4. Gunst SJ, Zhang W (2008) Actin cytoskeletal dynamics in smooth muscle: a new paradigm for the regulation of smooth muscle contraction. Am J Physiol Cell Physiol 295(3):C576-C587

5. Charras G, Paluch E (2008) Blebs lead the way: how to migrate without lamellipodia. Nat Rev Mol Cell Biol 9(9):730-736

6. Sedzinski J, Biro M, Oswald A, Tinevez JY, Salbreux G, Paluch E (2011) Polar actomyosin contractility destabilizes the position of the cytokinetic furrow. Nature 476(7361):462-466

7. Salbreux G, Charras G, Paluch E (2012) Actin cortex mechanics and cellular morphogenesis. Trends Cell Biol 22(10):536-545

8. Reymann AC, Boujemaa-Paterski R, Martiel JL, Guerin C, Cao W, Chin HF, De La Cruz EM, Thery M, Blanchoin L (2012) Actin network architecture can determine myosin motor activity. Science 336(6086):1310-1314

9. Bendix PM, Koenderink GH, Cuvelier D, Dogic Z, Koeleman BN, Brieher WM, Field CM, Mahadevan L, Weitz DA (2008) A quantitative analysis of contractility in active cytoskeletal protein networks. Biophys J 94(8):3126-3136

10. Kohler S, Schaller V, Bausch AR (2011) Collective dynamics of active cytoskeletal networks. Plos One 6(8):e23798

11. Kohler S, Schaller V, Bausch AR (2011) Structure formation in active networks. Nat Mater 10(6):462-468

12. Kohler S, Bausch AR (2012) Contraction mechanisms in composite active actin networks. Plos One 7(7):e39869

13. Murrell MP, Gardel ML (2014) Actomyosin sliding is attenuated in contractile biomimetic cortices. Mol Biol Cell 25(12):1845-1853

14. Schaller V, Schmoller KM, Karakose E, Hammerich B, Maier M, Bausch AR (2013) Crosslinking proteins modulate the selforganization of driven systems. Soft Matter 9(30):7229-7233

15. Murrell MP, Gardel ML (2012) F-actin buckling coordinates contractility and severing in a biomimetic actomyosin cortex. Proc Natl Acad Sci USA 109(51):20820-20825

16. e Silva MS, Depken M, Stuhrmann B, Korsten M, MacKintosh FC, Koenderink GH (2011) Active multistage coarsening of actin networks driven by myosin motors. Proc Natl Acad Sci USA 108(23):9408-9413

17. Mizuno D, Tardin C, Schmidt CF, MacKintosh FC (2007) Nonequilibrium mechanics of active cytoskeletal networks. Science 315(5810):370-373

18. Koenderink GH, Dogic Z, Nakamura F, Bendix PM, MacKintosh FC, Hartwig JH, Stossel TP, Weitz DA (2009) An active biopolymer network controlled by molecular motors. Proc Natl Acad Sci USA 106(36):15192-15197

19. Wang SS, Wolynes PG (2012) Active contractility in actomyosin networks. Proc Natl Acad Sci USA 109(17):6446-6451 
20. Lenz M (2014) Geometrical origins of contractility in disordered actomyosin networks. Phys Rev X 4(4):041002

21. Dasanayake NL, Michalski PJ, Carlsson AE (2011) General mechanism of actomyosin contractility. Phys Rev Lett 107(11):118101

22. Inoue Y, Tsuda S, Nakagawa K, Hojo M, Adachi T (2011) Modeling myosin-dependent rearrangement and force generation in an actomyosin network. J Theor Biol 281(1):65-73

23. Alvarado J, Sheinman M, Sharma A, MacKintosh FC, Koenderink GH (2013) Molecular motors robustly drive active gels to a critically connected state. Nat Phys 9(9):591-597

24. Sheinman M, Broedersz CP, MacKintosh FC (2012) Actively stressed marginal networks. Phys Rev Lett 109(23):238101

25. Broedersz CP, MacKintosh FC (2011) Molecular motors stiffen non-affine semiflexible polymer networks. Soft Matter 7(7):31863191

26. Kim T (2015) Determinants of contractile forces generated in disorganized actomyosin bundles. Biomech Model Mechanobiol 14(2):345-355

27. Craig R, Megerman J (1977) Assembly of smooth muscle myosin into side-polar filaments. J Cell Biol 75(3):990-996

28. Ferrer JM, Lee H, Chen J, Pelz B, Nakamura F, Kamm RD, Lang MJ (2008) Measuring molecular rupture forces between single actin filaments and actin-binding proteins. Proc Natl Acad Sci USA 105(27):9221-9226

29. Tyska MJ, Dupuis DE, Guilford WH, Patlak JB, Waller GS, Trybus KM, Warshaw DM, Lowey S (1999) Two heads of myosin are better than one for generating force and motion. Proc Natl Acad Sci USA 96(8):4402-4407
30. Veigel C, Molloy JE, Schmitz S, Kendrick-Jones J (2003) Loaddependent kinetics of force production by smooth muscle myosin measured with optical tweezers. Nat Cell Biol 5(11):980-986

31. Uemura S, Higuchi H, Olivares AO, De La Cruz EM, Ishiwata S (2004) Mechanochemical coupling of two substeps in a single myosin V motor. Nat Struct Mol Biol 11(9):877-883

32. Kim T, Gardel ML, Munro E (2014) Determinants of fluidlike behavior and effective viscosity in cross-linked actin networks. Biophys J 106(3):526-534

33. Kim T, Hwang W, Lee H, Kamm RD (2009) Computational analysis of viscoelastic properties of crosslinked actin networks. Plos Comput Biol 5(7):e1000439

34. Kaufman LJ, Brangwynne CP, Kasza KE, Filippidi E, Gordon VD, Deisboeck TS, Weitz DA (2005) Glioma expansion in collagen I matrices: analyzing collagen concentration-dependent growth and motility patterns. Biophys J 89(1):635-650

35. Lieleg O, Claessens MM, Luan Y, Bausch AR (2008) Transient binding and dissipation in cross-linked actin networks. Phys Rev Lett 101(10):108101

36. Broedersz CP, Depken M, Yao NY, Pollak MR, Weitz DA, MacKintosh FC (2010) Cross-link-governed dynamics of biopolymer networks. Phys Rev Lett 105(23):238101 